\title{
Silencing of ANKRD12 circRNA induces molecular and functional changes associated with invasive phenotypes
}

Thasni Karedath ${ }^{1+}$, Ikhlak Ahmed ${ }^{1+}$, Wafa Al Ameri ${ }^{1}$, Fatima M. Al-Dasim¹, Simeon S. Andrews ${ }^{1}$, Samson Samuel ${ }^{3}$, Iman K. Al-Azwani ${ }^{2}$, Yasmin Ali Mohamoud ${ }^{2}$, Arash Rafii ${ }^{4}$ and Joel A. Malek ${ }^{1,2^{*}}$ (D)

\begin{abstract}
Background: Circular RNAs (circRNAs) that form through non-canonical backsplicing events of pre-mRNA transcripts are evolutionarily conserved and abundantly expressed across species. However, the functional relevance of circRNAs remains a topic of debate.

Methods: We identified one of the highly expressed circRNA (circANKRD12) in cancer cell lines and characterized it validated it by Sanger sequencing, Real-Time PCR. siRNA mediated silencing of the circular junction of circANKRD12 was followed by RNA Seq analysis of circANKRD12 silenced cells and control cells to identify the differentially regulated genes. A series of cell biology and molecular biology techniques (MTS assay, Migration analysis, 3D organotypic models, Real-Time PCR, Cell cycle analysis, Western blot analysis, and Seahorse Oxygen Consumption Rate analysis) were performed to elucidate the function, and underlying mechanisms involved in circANKRD12 silenced breast and ovarian cancer cells.

Results: In this study, we identified and characterized a circular RNA derived from Exon 2 and Exon 8 of the ANKRD12 gene, termed here as circANKRD12. We show that this circRNA is abundantly expressed in breast and ovarian cancers. The circANKRD12 is RNase $R$ resistant and predominantly localized in the cytoplasm in contrast to its source mRNA. We confirmed the expression of this circRNA across a variety of cancer cell lines and provided evidence for its functional relevance through downstream regulation of several tumor invasion genes. Silencing of circANKRD12 induces a strong phenotypic change by significantly regulating cell cycle, increasing invasion and migration and altering the metabolism in cancer cells. These results reveal the functional significance of circANKRD12 and provide evidence of a regulatory role for this circRNA in cancer progression.
\end{abstract}

Conclusions: Our study demonstrates the functional relevance of circANKRD12 in various cancer cell types and, based on its expression pattern, has the potential to become a new clinical biomarker.

Keywords: Circular RNA, RNA-seq, Breast cancer, siRNA, Cancer invasion, OCR, OXPHOS

\footnotetext{
* Correspondence: jom2042@qatar-med.cornell.edu

†Thasni Karedath and Ikhlak Ahmed contributed equally to this work.

'Department of Genetic Medicine, Weill Cornell Medicine-Qatar, Education

City, Qatar Foundation, P.O. Box No, 24144 Doha, Qatar

${ }^{2}$ Genomics Core, Weill Cornell Medicine-Qatar, Education City, Qatar

Foundation, Doha, Qatar

Full list of author information is available at the end of the article
}

(c) The Author(s). 2019 Open Access This article is distributed under the terms of the Creative Commons Attribution 4.0 International License (http://creativecommons.org/licenses/by/4.0/), which permits unrestricted use, distribution, and reproduction in any medium, provided you give appropriate credit to the original author(s) and the source, provide a link to the Creative Commons license, and indicate if changes were made. The Creative Commons Public Domain Dedication waiver (http://creativecommons.org/publicdomain/zero/1.0/) applies to the data made available in this article, unless otherwise stated. 


\section{Background}

Exonic circular RNAs (circRNAs) are a class of RNA in biological systems for which function is not well understood. They possess distinct properties compared to linear RNAs and arise from direct backsplicing events that covalently link the $3^{\prime}$ end of an exon with the $5^{\prime}$ end of either the same exon or any other further upstream exon [1].

circRNAs were initially considered as molecular artifacts of aberrant RNA splicing [2]. This hypothesis was challenged by the observation that circRNAs are detected in various cell types in an evolutionarily conserved manner [3]. The copy number of circRNAs can be up to 10 times greater than that of associated linear RNAs, suggesting that these circRNAs may possess biological functions [4]. Studies have shown that some circRNAs harbor multiple binding sites for microRNAs, thereby "sponging" microRNAs and serving as competitive inhibitors for microRNA functions [5]. To serve as a sponge, however, a circular RNA must contain multiple microRNA-binding sites and be expressed at sufficiently high levels in the cytoplasm [1]. The majority of circRNAs may not fit this category, and their functions remain uncertain. Some circRNAs have been shown to interact with RNA binding proteins to form RNA protein complexes thereby regulating canonical linear splicing of the gene [6]. These findings suggest that circRNAs hold a dynamic and distinct role in gene regulation.

Emerging evidence suggests that regulation of circRNAs is closely associated with different diseases, particularly cancer with aberrant expression pattern [7-10]. Thus, circRNAs represent a new class of diagnostic biomarkers with potential therapeutic significance $[11,12]$. The longer half-lives compared to their linear counterparts makes circRNAs long-acting regulators of cellular behavior and robust biomarkers [13, 14]. A growing body of evidence has implicated the functional involvement of circRNAs in regulating cancer progression and proliferation [14-17].

In the present study, we investigated the functional role of a high abundance circRNA from Ankyrin Repeat Domain 12 (ANKRD12) gene in cancer progression. ANKRD12 is a paralog of ANKRD11, a putative tumor suppressor gene with multiple functions including as a p53 co-activator $[18,19]$. Low ANKRD12 level is an independent prognostic predictor of colorectal carcinoma patients [20]. Circular isoforms of ANKRD12 have been identified in cancer cells and patient samples in many recent studies including ours [21, 22]. In this study, we validated one of the most predominant circular isoforms of ANKRD12 gene that includes the backsplice junction of exons 8 and 2 (circANKRD12). We report that circANKRD12 regulates the invasion, migration proliferation and cellular bioenergetics of cancer cells by modulating cell signaling, metabolic and cell cycle regulation pathways in cancer. We confirmed the expression of this circRNA across a variety of cancer cell lines and provided evidence for its functional relevance through downstream regulation of several tumor invasion genes.

\section{Methods}

\section{Cell lines and treatment}

Ovarian cancer cell lines PA-1 (ATCC ${ }^{\oplus}$ CRL-1572), SKOV3(ATCC HTB-77 $^{\mathrm{Tm}}$ ),Caov3(ATCC ${ }^{\oplus} \mathrm{HTB}^{-75^{\mathrm{TM}}}$ ), NIH: OVCAR-3 (ATCC ${ }^{\bullet} \mathrm{HTB}^{-161^{\mathrm{T}}}$ ), breast cancer cell lines MDA-MB-231(ATCC ${ }^{\oplus}$ HTB-26 $^{\mathrm{m}}$ ), MCF7(ATCC ${ }^{\oplus}$ HTB- $^{-}$ $\left.22^{\mathrm{rm}}\right), \mathrm{T}^{-47 D}\left(\mathrm{ATCC}^{\ominus} \mathrm{HTB}^{-133^{\mathrm{rm}}}\right.$ ) and breast normal cell line MCF 10A $\left(\mathrm{ATCC}^{\ominus} \mathrm{CRL}^{-10317^{\mathrm{TM}}}\right)$, Lung cancer cell lines, HCC2935(ATCC ${ }^{\bullet}$ CRL-2869 $^{\mathrm{mm}}$ ) and NCI-H226 (ATCC ${ }^{\oplus}$ CRL-5826 ${ }^{\mathrm{TM}}$ ) and Lung Normal Fibroblast cell line LL 24(ATCC ${ }^{\circledR} \mathrm{CCL}^{-151^{\mathrm{TM}}}$ ) (all from American type Cell Collection, Manassas, VA), APOCC (ovarian primary cell line derived from ascites fluid) (pers. communication Dr. Arash Tabrizi), A27809 (93112519-1VL, Sigma), A2780 CIS (93112519-1VL,Sigma) were used for the current study. Cells were cultured in DMEM (Life Technologies, NY, USA) supplemented with $10 \%$ fetal bovine serum (Life Technologies, USA). Low Passage number cells were used for all the experiments. Cell culture were routinely checked for mycoplasma contamination using MycoAlert Mycoplasma detection kit (Lonza, Basel, Switzerland).

\section{RNA preparation and qRT-PCR}

The nuclear and cytoplasmic RNA was extracted using The SurePrep ${ }^{\mathrm{sm}}$ Nuclear or Cytoplasmic RNA Purification Kit (Fisher Grand Island, NY, USA). Total RNA from whole-cell lysates were isolated by using RNAesay mini kit (Qiagen Valencia, CA USA). For RNase R treatment, $2 \mu \mathrm{g}$ of total RNA was incubated $60 \mathrm{~min}$ at $40{ }^{\circ} \mathrm{C}$ with or without $3 \mathrm{U} \mathrm{\mu g}^{-1}$ of RNase $\mathrm{R}$ (Epicentre Technologies, Madison, WI), and the resulting RNA was subsequently purified using an RNeasy MinElute cleaning Kit (Qiagen, Valencia, CA USA). cDNA synthesis was carried out using First strand synthesis kit (AMV) from Roche Biosciences or Biorad select cDNA synthesis kit using random primer for circRNA experiments. Fast Start Universal SYBR Green Master mix (Roche, Clovis, CA) was used to amplify the specific gene using cDNA primes obtained from Primer bank (https://pga.mgh.harvard.edu/ primerbank/ (Additional file 4: Table S1). Each Real-Time assay was done in triplicate on Step One Plus Real-time PCR machine (Life Technologies, CA, USA).

\section{Transfection}

siRNA transfection was carried out using customdesigned siRNAs for both ANKRD12 circular and linear transcripts (Fig. 1 and Additional file 4: Table S1). The SKOV3, MDA-MB-231, OVCAR3, NCI-H226 cells were 


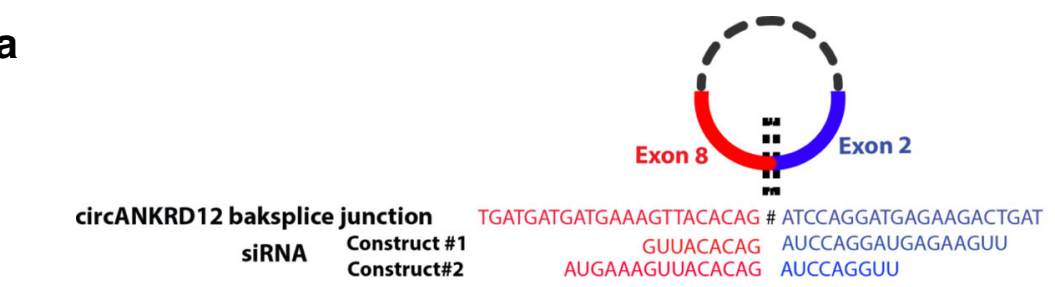

\section{b}

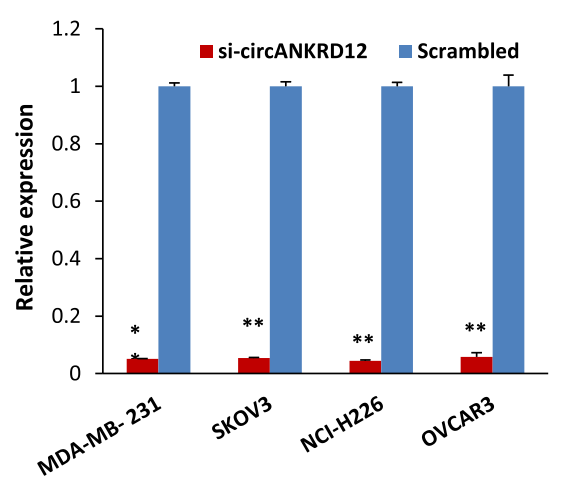

C

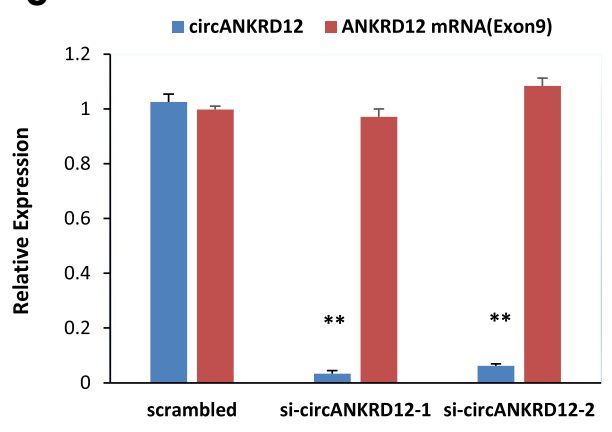

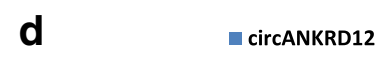

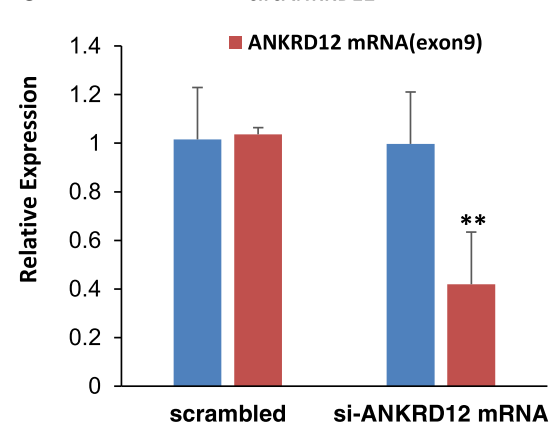

Fig. 1 siRNA mediated silencing of circANKRD12 in cancer cells a Two circANKRD12 siRNAs spanning the back-splice junction $\mathbf{b}$ qRT-PCR analysis for knockdown efficiency of circANKRD12 siRNA in 4 different cell lines. c qRT-PCR analysis for knockdown efficiency of two different circANKRD12 siRNA constructs in SKOV3 cells. $\mathbf{d}$ qRT-PCR analysis for silencing efficiency of ANKRD12 linear siRNA (exon9) in SKOV3 cells. (Data in b-d are the means with error bars indicating standard error of the mean (SEM) of three experiments. ${ }^{* *} P<0.01$ (Student's t-test)

grown in 6 well plates for transfection. The cells were transfected at $24 \mathrm{~h}$ with $30 \mathrm{pmol}$ concentration of siRNA (VWR, Radnor, PA, USA) or scrambled control (Mission siRNA universal negative control, Sigma, St.Louis, USA) using Lipofectamine RNAi max (Invitrogen MA USA) according to manufacturer's protocol. These experiments were conducted in three different biological triplicates for subsequent RNA-sequencing.

\section{RNAseq analysis}

SKOV3, OVCAR3, NCI-H226, MDA-MB-231 cells transfected with either circANKRD12 or a universal scrambled control were used for RNAseq analysis. RNAseq library preparation and In silico detection of circRNA candidates from paired-end RNA-seq data was conducted as described earlier [21].

\section{Cell proliferation assay}

Cells were cultured at a density of $5 \times 10^{3}$ cells per well in flat-bottomed 96-well plates in DMEM supplemented with $10 \%$ FBS with antimycotic antibiotic. The experiment was done at different time points $(24 \mathrm{~h}$ and $48 \mathrm{~h}$ or based on cell doubling time). CellTiter $96^{\circ}$ Aqueous One Solution Reagent (Promega, Madison, WI) was added and the experiment was conducted according to the manufacturer's instructions.

CellTiter-Glo assay (Promega Madison USA) for determining cell proliferation was conducted according to the manufacturer's instructions. Briefly, CellTiterGlo reagent was added directly to the wells of 96 well plate and luminescence was measured on an Envision reader (PerkinElmer). 
Scratch assay- cell migration assay

Scratch assay was conducted as described earlier [23]. Cells were plated into a 6-well plate with complete medium and grown to $80 \%$ confluence. Cells were transfected with respective siRNA in OPTIMEM medium, and the medium was replaced with serum-free DMEM after transfection. After cells were grown to $100 \%$ confluence, a wound was created by scraping the confluent monolayer cells with a p200 pipette tip. Cells were then grown either in serum-free medium or medium containing $3 \mathrm{mM}$ Thymidine. The distance between the two sides of the cell-free area was photographed using 10x objective in AXION Zeiss epifluorescence microscope. The distance is measured using Zeiss Zen software (Carl Zeiss Carpenteria, CA, USA).

\section{Trans-well migration and invasion assay}

Cellular migration and invasion were determined using a Transwell Boyden chamber assay as described previously [24].

\section{D organotypic spheroid model experiments}

3D anchorage-independent spheroids were developed in SKOV3 cancer cell lines; initially, cells were seeded on ultra-low attachment plate (Corning, NY, USA) for three days. Transfection of the spheroids with circANKRD12 siRNA was conducted using the reverse transfection method.

\section{Spheroid area measurement}

Cells were seeded at a density of 300,000 cells/well into ultralow attachment plates. After $72 \mathrm{~h}$, cells were transfected either with scrambled siRNA or circANKRD12 siRNA. After $48 \mathrm{~h}$ the diameters of at least 50 spheroids were measured and the spheroid area was measured Zeiss Zen Software.

\section{Cell proliferation assay in 3D organotypic models}

Ten thousand cells were seeded on each well of ultralow attachment 96 well plate with OPTIMEM medium. After three days, once spheroids were formed, cells were transfected with either circANKRD12 siRNA or scrambled control. After $24 \mathrm{~h}$ and $48 \mathrm{~h}$ of transfection, MTS reagent was added to the medium. Measurements were according to the manufacturer's instructions.

\section{Collagen invasion assay in 3D organotypic models}

$3 \mathrm{D}$ organotypic models of either circANKRD12 transfected or scrambled control in SKOV3 cells expressing GFP were placed on the top of jellified collagen matrix (Rat tail collagen $1,1 \mathrm{mg} / \mathrm{ml}$ ). The invasiveness analyzed after $24 \mathrm{~h}$ to 10 days.

\section{Cell cycle analysis}

Cell cycle analysis was done on cells fixed with FxCycle $^{\mathrm{Tm}} \mathrm{PI} / \mathrm{RNase}$ Staining Solution using BD LSRFortessa $^{\mathrm{TM}}$ cell analyzer.

\section{Western blot analysis}

Cellular protein was extracted after $48 \mathrm{~h}$ of transfection. The cells were lysed in 100ul of RIPA buffer with protease inhibitor cocktail. Then 40 micrograms of protein were resolved in SDS PAGE gel and transferred to a nitrocellulose membrane. The primary antibodies used were anti-Cyclin D1, Anti- Cyclin B1, Anti CyclinD2, anti-Cyclin Phospho B1 and $\beta$-actin (Cell Signaling, USA). The blots were visualized by ECL detection (Amersham, N J, United States). The Western blot experiments and analysis were done as described earlier [25]. Briefly, cell lysate protein $(25 \mu \mathrm{g})$ was separated on an SDS-Polyacrylamide gel and transferred onto nitrocellulose membranes. Membranes were then blocked with $5 \%(\mathrm{w} / \mathrm{v})$ non-fat dry milk tris-buffered saline (TBS) containing $0.1 \%(\mathrm{v} / \mathrm{v})$ Tween 20 and incubated at $4{ }^{\circ} \mathrm{C}$ with the primary antibody (1:1000 dilution). The excess primary antibody was washed off in TBS-T wash buffer, and the membranes were incubated with HRP-linked secondary antibodies (1:2500) for one $h$ at room temperature. The excess secondary antibody was then washed off in TBS-T, and the protein levels were detected using enhanced chemiluminescence reagent (Sigma-Aldrich, Inc., MO, USA) and imaged on a Geliance P600 gel documentation system (PerkinElmer, Inc. MA, USA). $\beta$-Actin was used as the loading control.

\section{circANKRD12 and ANKRD12 siRNA longevity assay}

siRNA longevity was calculated based on cell doubling time. The cell doubling time of SKOV3 cells was calculated based on the mathematical equation.

$$
\begin{aligned}
\text { Doubling Time } & =\operatorname{duration} * \log (2) \\
& / \log (\text { Final Concentration }) \\
& -\log (\text { Initial Concentration }) .
\end{aligned}
$$

Cells were seeded on $60 \mathrm{~mm}$ petri plates and transfection was done with siRNA of circANKRD12, or ANKRD12 mRNA. Cells were harvested on an interval of $48 \mathrm{~h}$ (doubling Time). The experiment was extended till 10th doubling time (20 days). The silencing efficacy was measured by gene expression analysis (qRT-PCR).

\section{Assessment of mitochondrial function by seahorse extracellular flux analyzer}

The mitochondrial oxygen consumption rate (OCR) in the MDA-MB-231 and SKOV3 cells was assessed by using a Seahorse Bioscience XFe96 analyzer (Massachusetts, 
USA). Seahorse Bioscience XF Cell Mito Stress Test assay kit was used for the study. In this assay, subsequent additions of the ATP synthase inhibitor oligomycin, the mitochondrial uncoupler carbonyl cyanide 4-(trifluoromethoxy) phenylhydrazone (FCCP) and the complex I + II inhibitors rotenone + antimycin A were injected into the assay medium containing cells with different treatments as per manufactures protocol. 10, 000 cells were seeded on assay plate and the assay was completed in $48 \mathrm{~h}$ after the transfection.

\section{Statistical analysis}

Statistical analysis in all the experiments is based on at least three biological replicates and the error bars are drawn with the standard error of means (SEM). The $p$ value is calculated by using 1-tailed student's $\mathrm{T}$ test.

\section{Data access}

The RNA-seq data for cell-lines has been submitted to Sequence Read Archive (NCBI SRA) under Bio project accession number PRJNA526399.

SRA records will be accessible with the following link: https://www.ncbi.nlm.nih.gov/sra/PRJNA526399

\section{Results}

\section{Characterization of circANKRD12 in cancer cells}

We characterized the circular RNA expression using RNA sequencing (RNA-seq) of total RNA in ovarian cancer cell lines (SKOV3 and CAOV3) undergoing Epithelial to Mesenchymal Transition. Using our circRNA detection pipeline [21], we identified a total of 18,700 circRNAs in these cell lines. The circRNA from the ANKRD12 gene having a backsplice junction between exons 8 and 2 (circANKRD12) was one of the highly abundant circular RNAs in both SKOV3 and CAOV3 cell lines. The circANKRD12 originates from 18p11.22 chromosome locus with the backsplice junction forming between exons located $\sim 39.3 \mathrm{~kb}$ apart (Fig. 2a). By carefully designing two sets of divergent primer pairs in Exon2 and Exon8 and Sanger sequencing of the amplified products, we were able to identify two circular isoforms of $286 \mathrm{bp}$ and $925 \mathrm{bp}$ lengths sharing the same backsplice junction (Additional file 3: S2:1-3), Additional file 4: Table S2). The first isoform of $286 \mathrm{bp}$ is comprised of only two exons (Exon2 and Exon8), while the 925 bp isoform is comprised of 6 exons (Exons 2,3,5, 6,7 and 8). All subsequent functional studies do not distinguish between these two circular isoforms as they targeted the junction sequence common to both circular forms but distinct vis-à-vis linear forms.

Multiple validation experiments were used to confirm circANKRD12 expression (Fig. 2b-d). Divergent primers were designed to amplify the backsplice exon junction. As expected, each primer pair produced a single distinct band of expected product size in PCR assay indicating the presence of the circular junction. The backsplice junctional sequence was confirmed by Sanger sequencing. The divergent primers, with respect to the genomic sequence, only amplified when cDNA was used as a template. The same primers did not produce a product from genomic DNA (gDNA). Conversely, PCR using convergent primers with respect to genomic sequence could amplify both cDNA and gDNA templates from ANKRD12 gene (Fig. 2b). This strongly indicates that a head-to-tail backsplicing junction in circANKRD12 only exists in the RNA form.

To examine whether circANKRD12 is resistant to exonucleases, we treated the total RNA extracted from the SKOV3 cell line with RNase R. This exoribonuclease enzyme digests all linear RNA forms with a 3 ' singlestranded region of greater than 7 nucleotides [26]. As circRNAs are devoid of any 3' single strand overhangs, they are expected to show resistance to digestion by RNase $R$. Indeed, circANKRD12 was resistant to RNase R digestion compared to linear forms of ANKRD12, HIF1 alpha, and GAPDH. Resistance to digestion with RNase $R$ exonuclease confirmed that the circANKRD12 is a stable circularized transcript (Fig. 2c). Further, cDNA created by priming with oligo (dT) primers failed to produce any amplification products for circANKRD12 in the PCR assay confirming the circular form does not have a typical polyA tail as do linear mRNAs. On the other hand, priming with random hexamers - which can also amplify non-polyadenylated RNAs - resulted in distinct PCR bands for the backsplice junction (Fig. 2d). The PCR and qRT-PCR analysis of nuclear and cytoplasmic fractions of RNA demonstrated that ANKRD12 circRNA is predominantly localized in the cytoplasm (Additional file 1: Figure S1:Fig. 2a, b). The purity of cytoplasmic and nuclear fractions was confirmed by amplifying the fractions using nuclear and cytoplasmic specific markers (Additional file 1: Figure S1: Fig a, b, Additional file 3: S2:8).

We performed Real-time PCR analysis on 12 different cell lines from breast, ovarian and lung cancer and normal breast and lung to assess the cell-type specific expression of circANKRD12 (Additional file 1: Figure S1:Fig. 2c). Most of these cell lines show a high abundance of both ANKRD12 circRNA and mRNA. Ovarian cancer cell lines show a higher abundance of ANKRD12 circRNA compared to breast and lung cancer cell lines.

\section{siRNA-mediated knockdown of circANKRD12 is highly specific}

To investigate the role of circANKRD12, we designed siRNAs to target the backsplice junction (Fig. 1a) and transfected multiple cancer cell lines to induce siRNAmediated knockdown of the circle while leaving the linear RNA unaffected. The circRNA specific siRNA was 
a
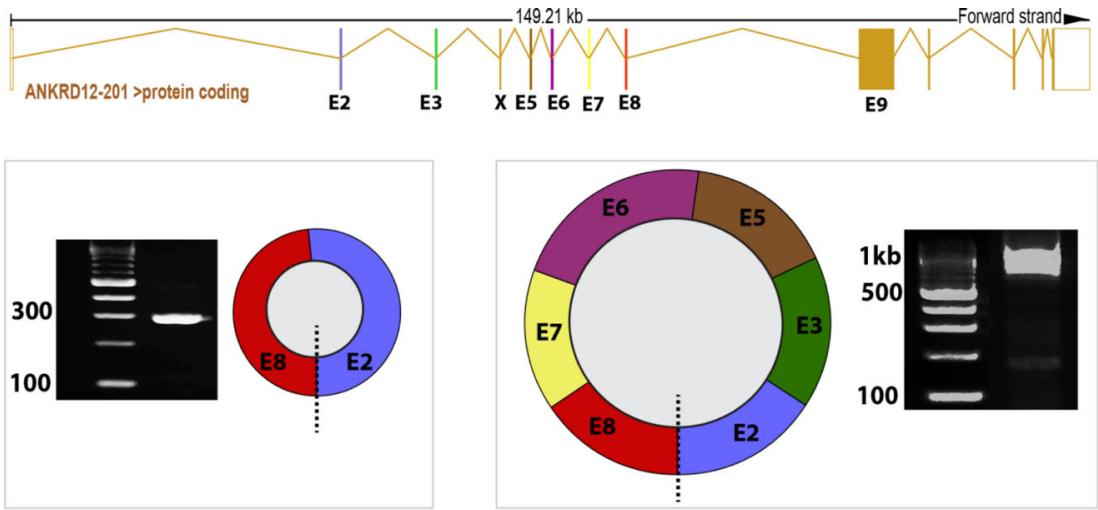

EXON 8

EXON 2

A A A GT TACACA G:ATC CA G G AT G A
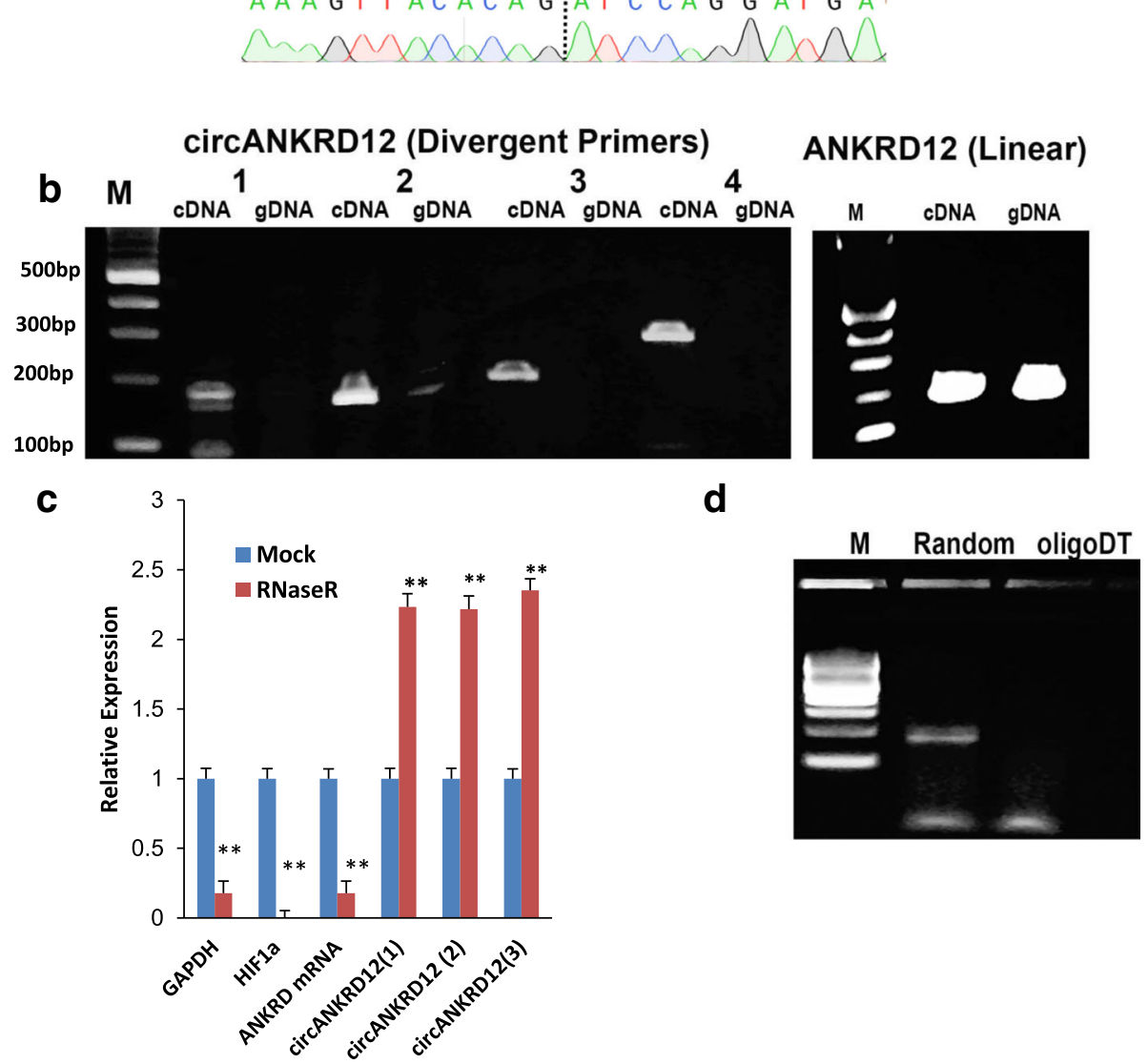

Fig. 2 Characterization of circANKRD12 in human cancer cells. a Transcript structure of the ANKRD12 gene. Two predominant circular isoforms (286 bp and 925 bp) were detected sharing the same backsplice junction between Exon8 and Exon2 as represented by the dashed line. The backsplice junction was sequenced for validation. b Divergent primers designed to detect the circANKRD12 backsplice junction amplified cDNA but not genomic DNA (4 pairs of different primers were used to amplify the circular junction). Linear ANKRD12 amplicon was detected in both CDNA and gDNA. M represents 100 bp ladder. c qRT-PCR for relative abundance of circANKRD12 and linear ANKRD12, GAPDH and HIF1 alpha in SKOV3 cells treated with RNase R $\mathbf{d}$ Backsplice junction was detected in CDNA synthesized using random hexamer but not oligoDT primed RNA. The data in $\mathbf{c}$ is means with error bars representing standard error of the mean (SEM) from three experiments; ${ }^{* *} P<0.01$ (Student's t-test)

designed against the backsplice junction spanning exons 2 and 8 of the gene. We observed high knockdown efficiency of greater than $90 \%$ of the circular junction when using the siRNA versus the control (scrambled siRNA) in four cell-line transfections (Fig. 1b). Using two siRNA constructs against the circANKRD12, we confirmed that knockdown of the circular RNA is specific and has no significant effect on the linear 
mRNA expression (Fig. 1c). The two different siRNAs had similar results reducing the likelihood that the observed effects are due to off-target knockdown. We also designed siRNAs targeting exon9 and exon7 of ANKRD12 gene to knockdown the linear mRNA. Indeed, the siRNA designed against exon 9, which lies outside the circANKRD12 locus was successful at knocking down the mRNA and exhibits no effect on circANKRD12 levels (Fig. 1d). However, the siRNA designed on Exon7 which is shared between mRNA and one of the circANKRD12 isoforms shows a remarkable reduction in mRNA levels as well a minimal but significant reduction in circANKRD12 levels. We investigated the specificity of siRNA constructs designed against circANKRD12, Exon7, and Exon9 through a series of knockdown experiments explained in Additional file 4: Supplementary file S2:4-7).

\section{Silencing of circANKRD12 changes molecular phenotypes} of ovarian, breast and lung cancer cells

RNA-sequencing was performed in triplicate for two ovarian cancer cell lines (SKOV3, OVCAR3), breast (MDAMB-231) and a lung cancer cell line (NCI-H226) for both scrambled control and siRNA targeting circANKRD12. Table 1 gives the number of differentially expressed genes in circANKRD12 knockdown samples (Additional file 5: Supplementary file S1) at a false discovery rate of less than $5 \%$ with at least 1.5 -fold changes in expression.

The canonical pathways analysis by Ingenuity's IPA toolkit (IPA ${ }^{\circ}$, QIAGEN Redwood City, (https://www.qiagenbioinformatics.com/products/ingenuity-pathway-analysis/) revealed an enrichment of differentially regulated genes involved in cell cycle regulation, invasion, migration, and interferon signaling pathways (Fig. 3, Additional file 2: Figure S2, Additional file 4: Table S3). We observed an upregulation of inflammatory pathways such as tumor necrosis pathway, NFkB pathway, interferon signaling and down regulation of the cell cycle pathway (Additional file 3: S2:15). Silencing of circANKRD12 affects cellular bioenergetics by downregulation of key genes involved in oxidative phosphorylation, AMPK pathway and cell metabolism (Additional file 4: Table S4). IPA predicts a significant activation of interferon signaling pathway through upregulation of STAT1, STAT2, IFT1, MX1genes, and downregulation of

Table 1 Number differentially up and downregulated genes for four cancer cell lines in siRNA-mediated knockdown of circANKRD12

\begin{tabular}{lcllll}
\hline & \multicolumn{5}{c}{ Si-circANKRD12 vs. Scrambled } \\
\cline { 2 - 5 } & SKOV3231 & OVCAR3 & MDA-MB-231 & NCI-H226 \\
\hline Upregulated genes & 25 & 104 & 237 & 168 \\
Downregulated genes & 9 & 71 & 179 & 3 \\
\hline
\end{tabular}

estrogen mediated $\mathrm{S}$ phase entry through downregulating cyclin D1 and cyclin A. Activation of IL8 signaling and inflamasome pathways, closely associated with immune modulation is also predicted. IL8 signaling pathway upregulates IL8, IRAK, ICAM-1, COX-2, and VEGF genes there by upregulating angiogenesis, inflammation, and inhibits cell proliferation by downregulating cyclin D1. A heatmap of differentially expressed genes from RNAseq analysis shows consistent gene expression changes in circANKRD12 silenced cells in cancer cell lines (Fig. 4a). The differentially regulated genes cyclin D1 (CCND1), CBX5, STAU1, and AK4 were found to be consistently downregulated in the circANKRD12 knockdown across multiple cell lines (Fig. 4a). Using qRTPCR, we validated the differential expression of these selected genes in SKOV3 cells (Fig. 4c). Cyclin D1 (CCND1) was among the genes that showed consistent downregulation in the circANKRD12 knockdown across multiple cell lines (Fig. 4a). To ensure that the effect on CCND1 is related to circANKRD12 knockdown rather than off-target effects we compared the knockdown effects in SKOV3 and LL24 cells (Fig. 4b). Unlike SKOV3 cells, the circANKRD12 level is low in LL24 cell line, attempts at knocking down circANKRD12 should not affect the cyclin D1 level in the cell line. Indeed, transfection of the siRNA against circANKRD12 in LL24 showed a reduction of the already low levels of circANKRD12 but no effect on cyclin D1 expression, suggesting the siRNA effect on CCND1 is likely mediated through circANKRD12. Because cell cycle regulation seems to be one of the most deregulated pathways in circANKRD12 silenced cells, we decided to follow it up with further functional screening using cell based phenotypic assays.

\section{siRNA mediated silencing of circANKRD12 increases migration and invasion in ovarian cancer cells}

Wound healing assays show that silencing of cirANKRD12 increased cell migration (Fig. 5a, b, Additional file 3: S2:10), in SKOV3 cells after $24 \mathrm{~h}$ and $48 \mathrm{~h}$. compared to scrambled control. This is confirmed again by cell migration assays in synchronized (Thymidine incorporation) cells, which show an increased migration rate for circANKRD12 silenced SKOV3 cells (Fig. 5c). Matrigel invasion (inserts coated with matrigel) and migration analysis using Boyden chamber shows circANKRD12 silenced cells undergo significant increase in the invasion and migration compared to scrambled control (Additional file 3: S2:10).

\section{Silencing of circANKRD12 decreases cell proliferation} Knockdown of circANKRD12 and ANKRD12 mRNA significantly reduces cell proliferation (Fig. 5d, Additional file 3: S2:9). The results of MTS and ATP assays show a significant reduction in cell proliferation in circANKRD12 
a

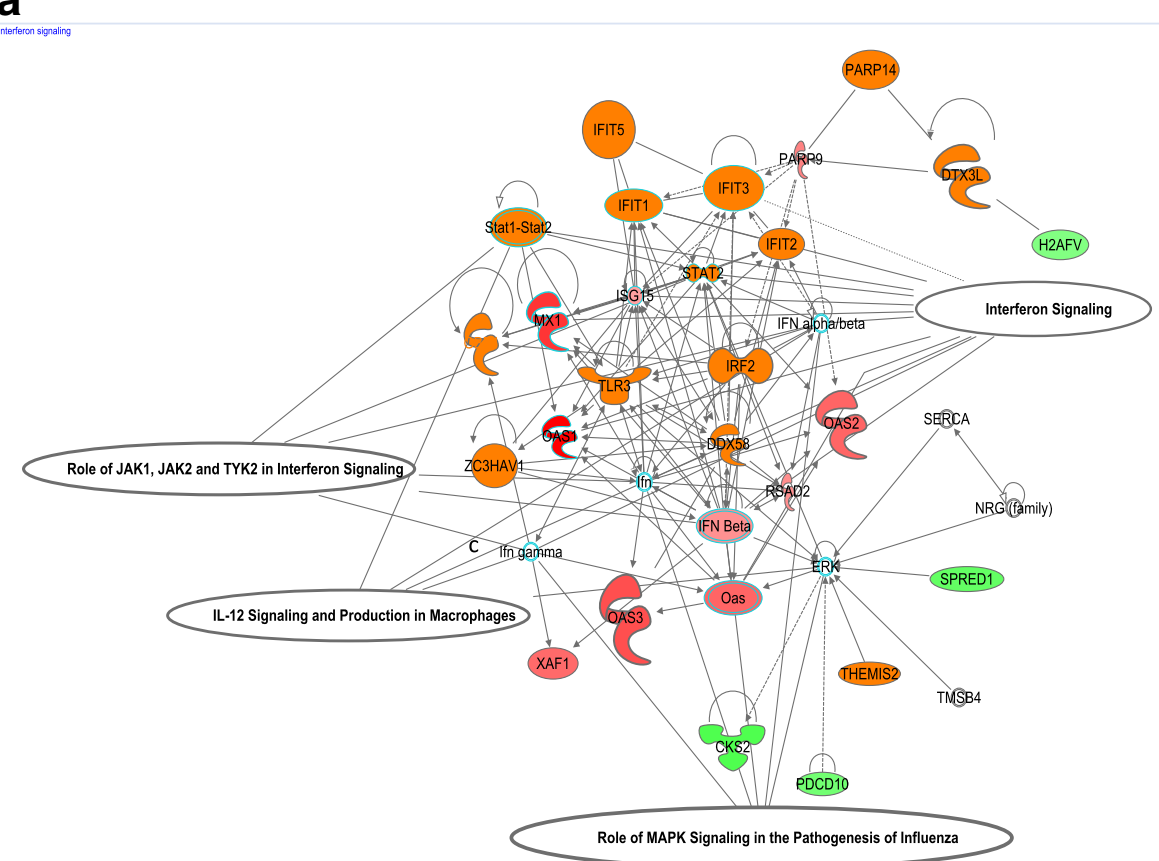

b

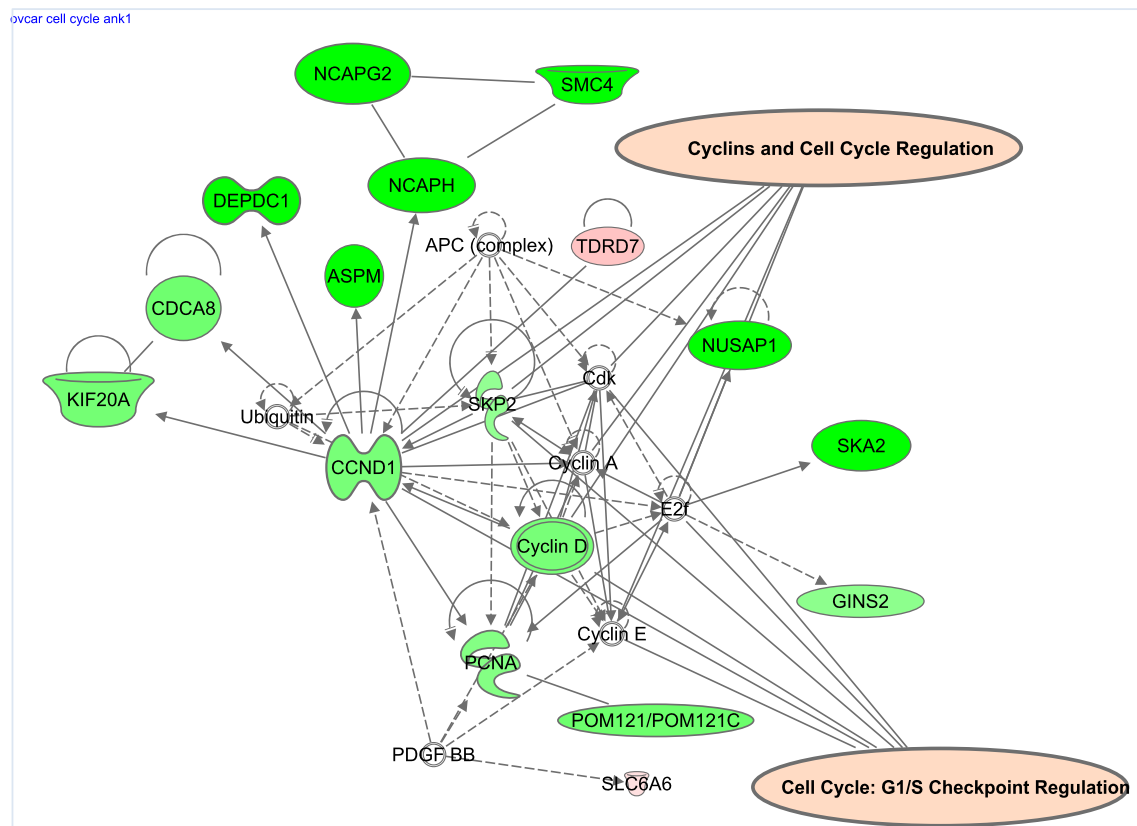

192000-2017 QAAGEN. Al rights reserved

Fig. 3 Gene network analysis of RNA-Seq data from MDA-MB-231 and OVCAR3 cells. a Gene network analysis using ingenuity pathway analysis in MDA-MB-231 cells silenced for circANKRD12 shows upregulation of Interferon, JAK/STAT, IL-12 signaling pathways. $\mathbf{b}$ Gene network analysis using ingenuity pathway analysis in OVCAR3 cells silenced with circANKRD12 shows downregulation of Cyclins and Cell Cycle regulation pathways

silenced cells compared to scrambled control (Fig. 5d, e. The Trypan blue exclusion assays show the silencing of circANKRD12 is not affecting the cell viability significantly. However, silencing of ANKRD12 mRNA significantly reduces both proliferation and cell viability in
SKOV3 cells (Fig. 5f). These results indicate that knockdowns of both circular and linear RNA forms of ANKRD12 gene are capable of inducing strong phenotypic changes and modulate the growth or survival of cancer cells. 
a

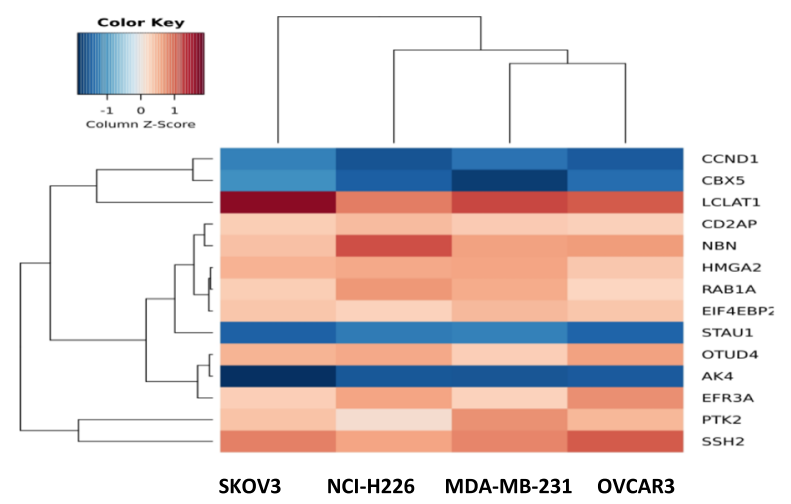

b
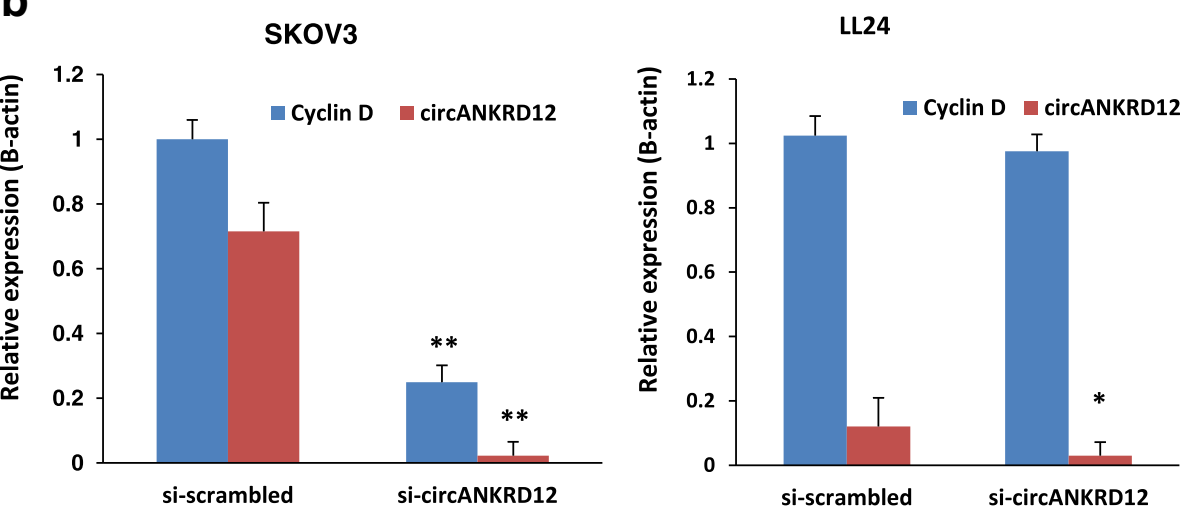

C

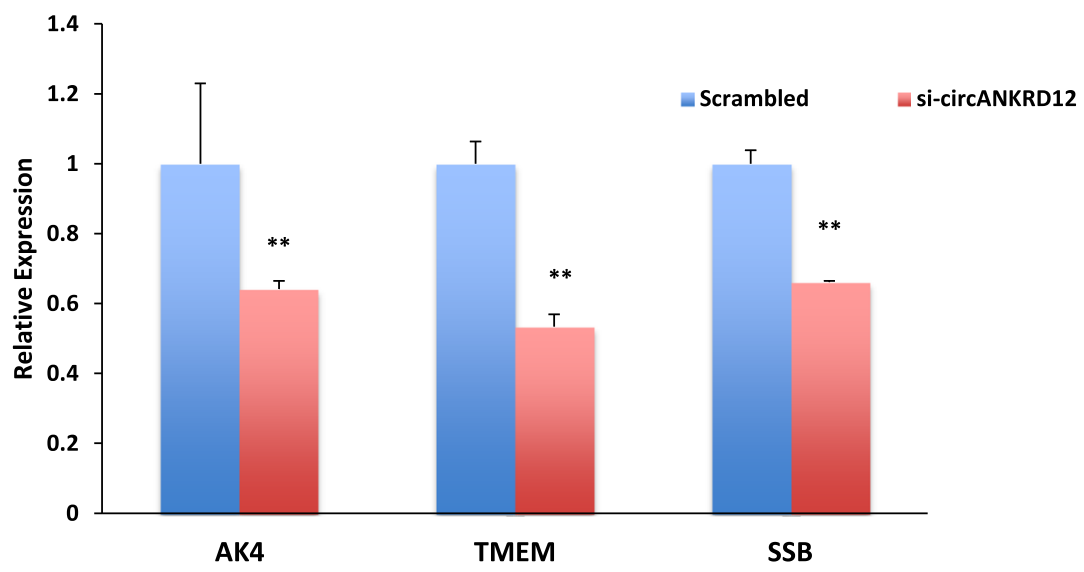

Fig. 4 Silencing of circANKRD12 affects gene expression changes in cancer cells. a Heat map of differentially expressed genes from RNAseq analysis of SKOV3, NCl-H226, MDAMB 231 and OVCAR3 cells silenced with si-circANKRD12 compared to scrambled control. b qRT-PCR validation of expression level of cyclin D1 in circANKRD12 silenced SKOV3 and LL24 cell lines. In SKOV3 cells, circANKRD12 knockdown leads to a significant downregulation of Cyclin D1 ( $P=7.8 \mathrm{E}-04)$, while as in LL24 Cyclin D1 shows a minimal non-significant change in expression $(P=0.18)$. c qRT-PCR validation of some selected genes differentially expressed in SKOV3 cells silenced with circANKRD12. Data in $\mathbf{b}-\mathbf{c}$ are the means with error bars indicating standard error of the mean of three experiments. ${ }^{* *} P<0.01$ (Student's t-test)

Silencing of cirANKRD12 in 3D tumor models induces a phenotypic switch from highly proliferative to an invasive phenotype

Since 3D culture is known to have better cell-to-cell interactions and more closely mimics tumors in vivo
[27], we investigated the effects of circANKRD12 knockdown in $3 \mathrm{D}$ culture experiments. The $3 \mathrm{D}$ anchorage independent growth of siRNA transfected SKOV3 cells showed smaller, dense aggregates associated with an invasive phenotype (Additional file 3: S2:12). The 3D 


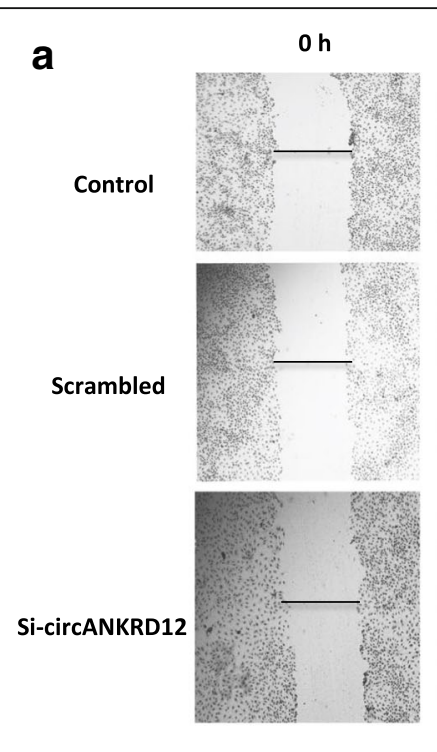

C
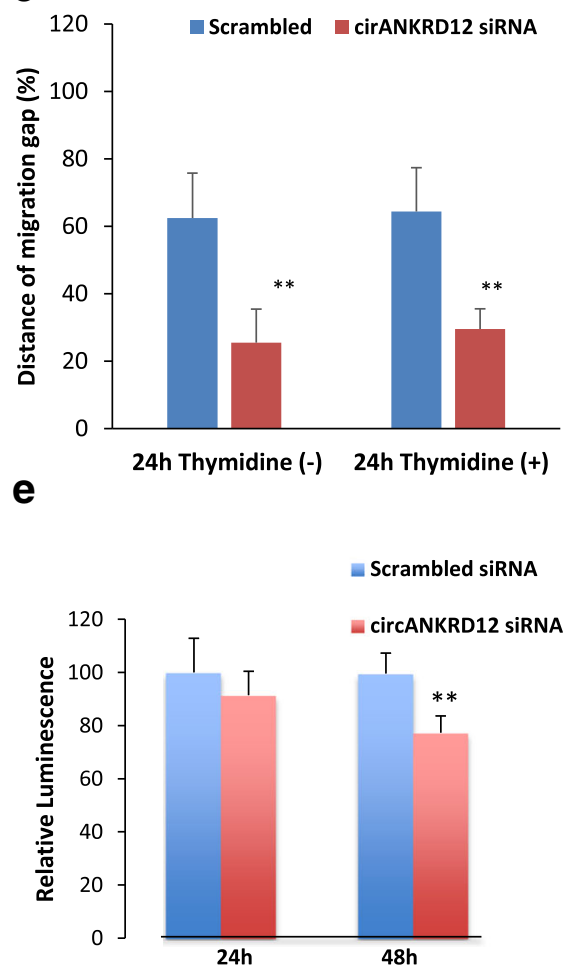

b
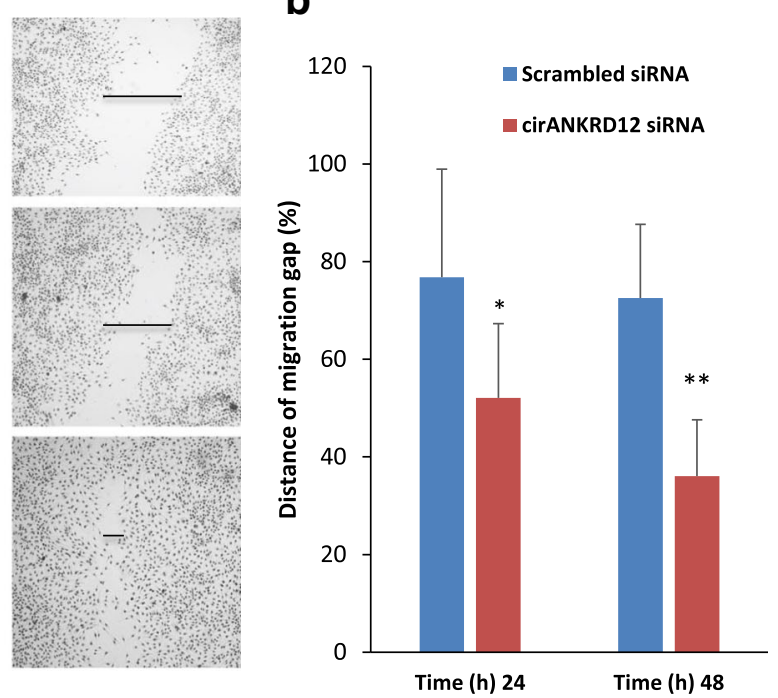

d
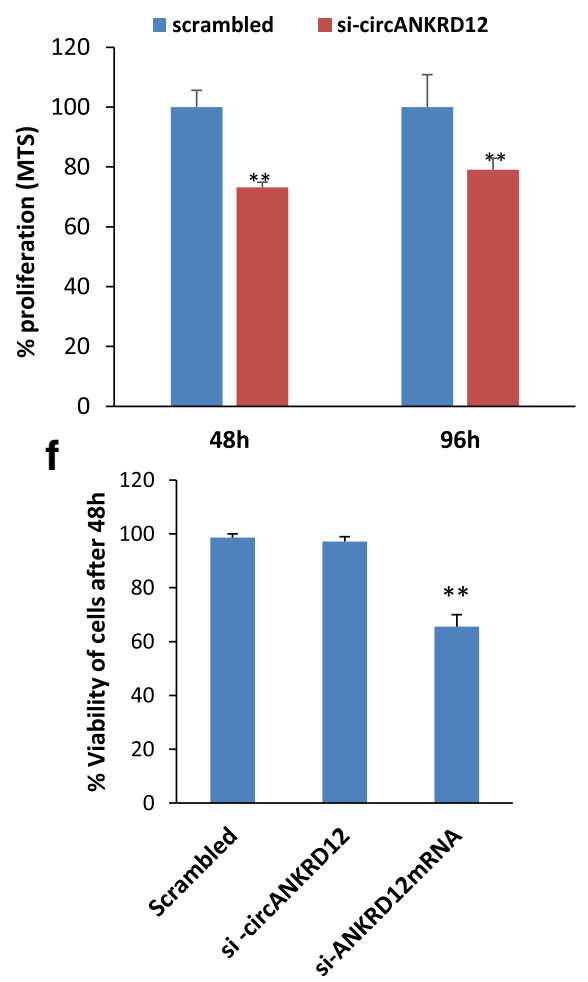

Fig. 5 Silencing of circANKRD12 results in an invasive phenotype with low proliferation rate. a Representative Wound healing migration assay image of circANKRD12 silenced SKOV3 cells and its scrambled control at $0 \mathrm{~h}$ and $24 \mathrm{~h}$. $\mathbf{b}$ Bar diagram depicting the reduction in wound width after migration in scrambled and circANKRD12 silenced cells (average of 15 wounds from three different biological replicates). c Migration of circANKRD12 silenced SKOV3 cells after cell cycle synchronization with thymidine. $\mathbf{d}$ The proliferation of SKOV3 cells transfected with siRNA against circANKRD12 and assessed using MTS cell proliferation assay kit at the indicated hours and data shown as \% proliferation compared to control. e Cell titer glow assay shows relative luminescence rate of ATP production in circANKRD12 silenced SKOV3 cells compared to scrambled control. f Cell viability is measured by using trypan blue exclusion assay. In circANKRD12 knockdown, the viability is reduced by roughly $2 \%$ while it is reduced by $\sim 34 \%$ in ANKRD12-mRNA knockdown. (Data in $\mathbf{b}-\mathbf{f}$ are the means with error bars indicating standard error of the mean of three experiments. ${ }^{* *} P<0.01$ (Student's $t$-test) 
organotypic models were efficiently silenced with circANKRD12 siRNA with 74\% knockdown efficacy (Fig. 6a). This reduction in spheroid size in circANKRD12-silenced SKOV3 organotypic models results in an alteration of phenotype from less invasive to a more invasive one (Fig. 6b). Cell proliferation MTS assay shows a significant reduction in cell growth within a time period of $24 \mathrm{~h}$ and $48 \mathrm{~h}$ after knockdown of ANKRD12 circRNA (Fig. 6c). Collagen invasion of the circANKRD12 silenced spheroids shows increased invasion through the collagen gel with highly motile cells after 10 days of transfection (Fig. 6d). These results indicate the phenotypic switching from a highly proliferative phenotype to a less proliferative phenotype with high invasion potential.

Cyclin D1 is down-regulated in circANKRD12 silenced cells and involved in phenotypic switching by facilitating G1 arrest

circANKRD12 silencing results in cyclin D1 downregulation and subsequent invasion and reduction in proliferation in ovarian cancer SKOV3 cells. Both real-time PCR and RNA-sequencing showed down-regulation of cyclin D1 in circANKRD12 silenced cells. The qRT-PCR analysis shows cyclin D1 expression is downregulated at least until $48 \mathrm{~h}$ after transfection with the siRNA (Fig. 7a). This is also supported by the Western blot analysis, which shows a reduction in cyclin D1 protein level (Fig. 7b). Only cyclin D1 shows differential regulation at the protein level in circANKRD12 silenced cells compared to other cyclin members including cyclin E1, pB1, and D2 (Additional file 3: S2:13). To estimate the duration of the RNAi effect initiated by transfecting siRNA, we performed a longevity assay of circANKRD12 siRNA (si-circANKRD12). The longevity, initially stable for six days (Additional file 3: S2: 14) was checked on a cell doubling time basis estimated to be $48 \mathrm{~h}$ (see Methods). The si-circANKRD12 exhibits an extended longevity period in SKOV3 cells, approaching nine doublings (18 days). The reduction in the level of cyclin D1 is on par with the observed silencing efficiency and longevity of siRNA (Fig. 7c). After the 10th doubling, the effect on cell proliferation induced by silencing of circANKRD12 becomes insignificant (Fig. 7d), suggesting that the knockdown efficiency is lost due to multiple passages of cells. As cyclin D1 is involved in cell cycle progression [28], cell cycle analysis of circANKRD12 knockdowns was conducted. Cell cycle analysis using FACS shows there is a significant G0/G1 cell cycle arrest in SKOV3 cells silenced with circANKRD12 compared to scrambled control (Fig. 8a, b).

\section{Silencing of circANKRD12 affects oxygen consumption ratio (OCR) in SKOV3 and MDAMB231 cells}

As AMPK signaling and other metabolic pathways are affected in circANKRD12 knockdown (Additional file 4:
Table S4). In order to determine whether the altered gene expression of these pathways translates to changes in basic metabolism, we analyzed metabolic phenotypes of circANKRD12 silenced MDA-MB-231 and SKOV3 cells using Seahorse extracellular flux analyzer. The oxygen consumption ratio (OCR) and ATP linked OCR analysis shows that knockdown of circANKRD12 decreases oxidative phosphorylation (OXPHOS) of MDAMB231 cells and SKOV3 cells (Fig. 8c-e). Previous reports have suggested that high invasive potential of cancer cells is negatively correlated to high energetic cancer phenotype $[29,30]$. These results thus indicate that circANKRD12 silencing can induce phenotypic switching between highly proliferative cells to highly invasive cells through cyclin D1 deregulation and shifting the oncobioenergetics to a low energy phenotype to facilitate invasion.

\section{Discussion}

Circular RNAs are attracting greater attention in RNA biology as there is growing evidence for their role in gene expression regulation. Even though a large repertoire of circRNAs has been identified in different organisms, tissues, diseases, and developmental conditions, only a few have been evaluated for their role in cellular functions [31-34]. Functional screening using siRNA targeting is difficult for circRNAs compared to other RNA types as the choice of the target region is limited to a few base pairs with specificity only at the backsplice junction. This constraint severely restricts the scale of circRNAs suitable for further functional studies using siRNA-mediated knockdown approaches.

In this study, we identified and characterized circular RNA isoforms from ANKRD12 gene (circANKRD12) that are abundantly expressed in ovarian and breast cancer cells. A recent study identified stable levels of circANKRD12 (exon 2-8) in young and old erythrocyte cells [35]. We show that circANKRD12 is a stable circularized transcript, resistant to RNase $\mathrm{R}$ digestion and devoid of a polyA tail. In contrast to the linear mRNA form, which is predominantly nuclear, circANKRD12 is localized in the cytoplasm.

Differential gene expression analysis by RNA-seq of circANKRD12 silenced cells revealed that silencing may accelerate cancer cell invasion, migration, and cellular movement by regulating a cascade of genes involved in these processes (Additional file 4: Table S3, 5). Interferon signaling and cell cycle checkpoint regulation by G1/S transition are the top networks deregulated in circANKRD12-silenced cells. IPA analysis shows an increase in pathways related to the invasion of cancer cells with significant $p$-value and $\mathrm{z}$ score (Additional file 2: Figure S2: Fig. 2a,b,c) in MDA-MB-231 cells. The key genes upregulated in circANKRD12 silenced cells are 

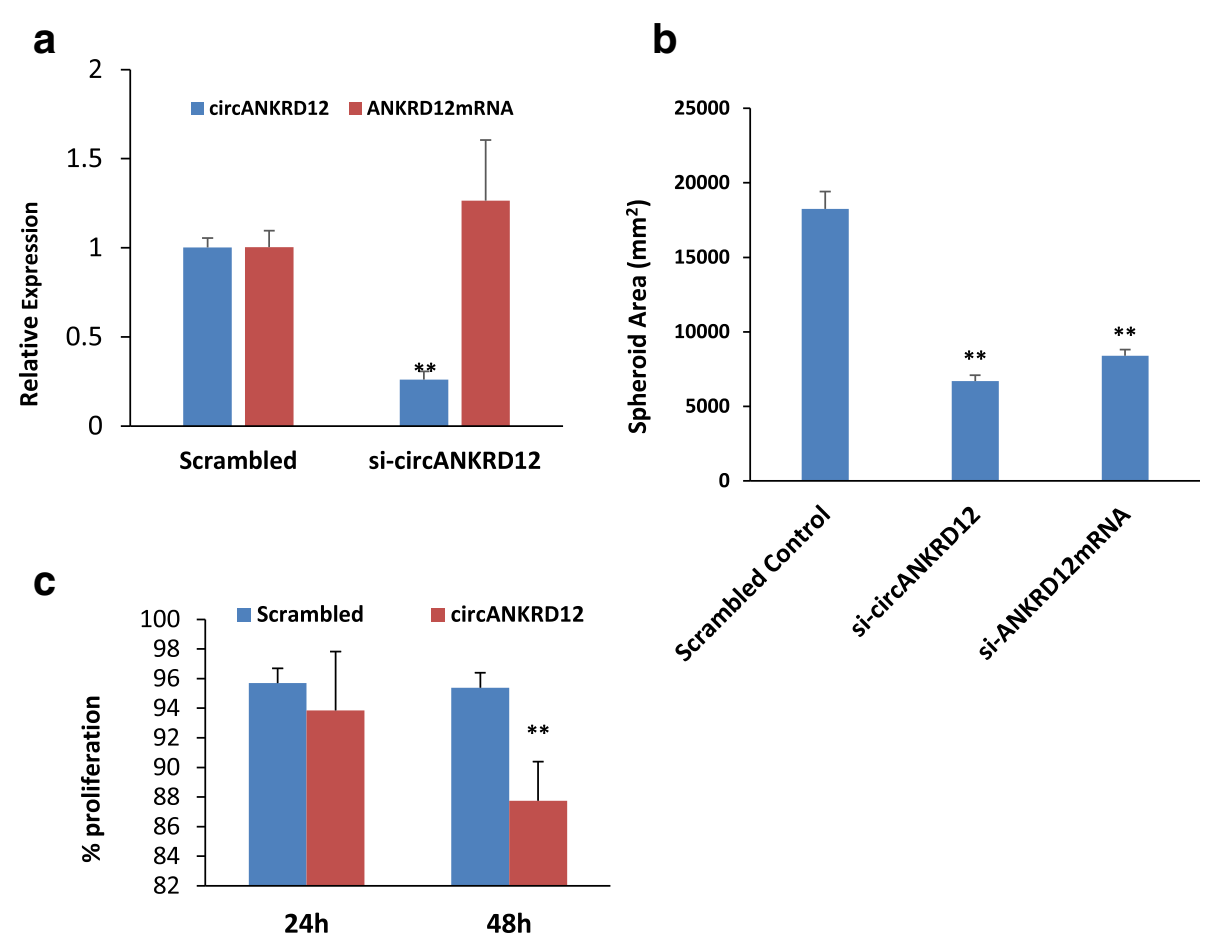

d

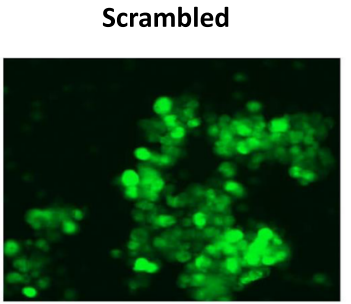

\section{Si-circANKRD12}

3D $-24 h$

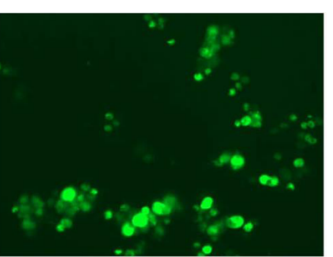

Collagen-48h
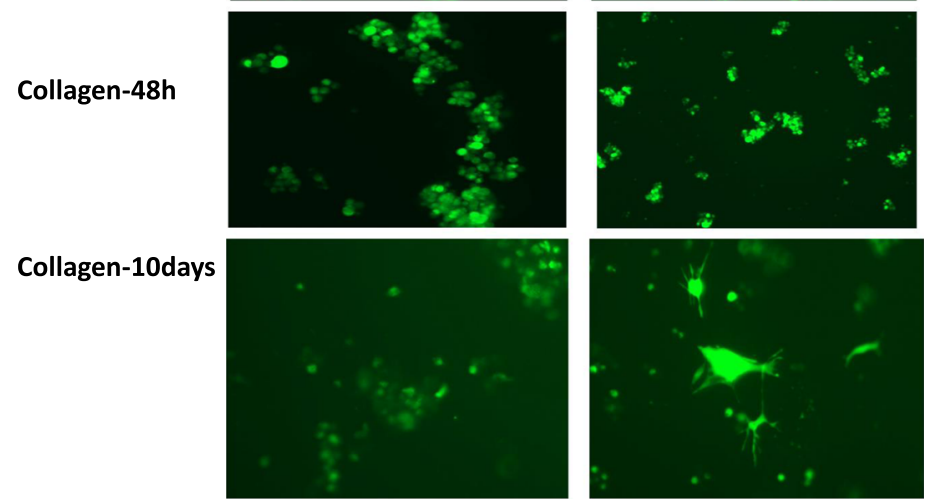

Fig. 6 Silencing of circANKRD12 in 3D spheroid models of SKOV3 cells shows an invasive phenotype. a Knockdown efficacy of circANKRD12 in 3D cultures. siRNA directed against circANKRD12 downregulates circular RNA but shows no downregulation on mRNA of ANKRD12 gene in SKOV3 organotypic models (b) spheroid area is measured in anchorage-independent 3D spheroids of SKOV3 cell lines, average of 100 spheroids in 3 independent experiments were measured. c MTS assay shows cell viability in 3D organotypic models is reduced at 24 and $48 \mathrm{~h}$ of transfection. $\mathbf{d}$ Collagen invasion assay of spheroids $24,48 \mathrm{~h}$ and 10 days. circANKRD12 silenced cells were able to invade through the thick collagen matrix and circANKRD12 silenced cells shows an invasive phenotype. Data in a-c are the means with error bars representing standard error of the mean of three experiments. ${ }^{*} P<0.01$ (Student's $t$-test) 


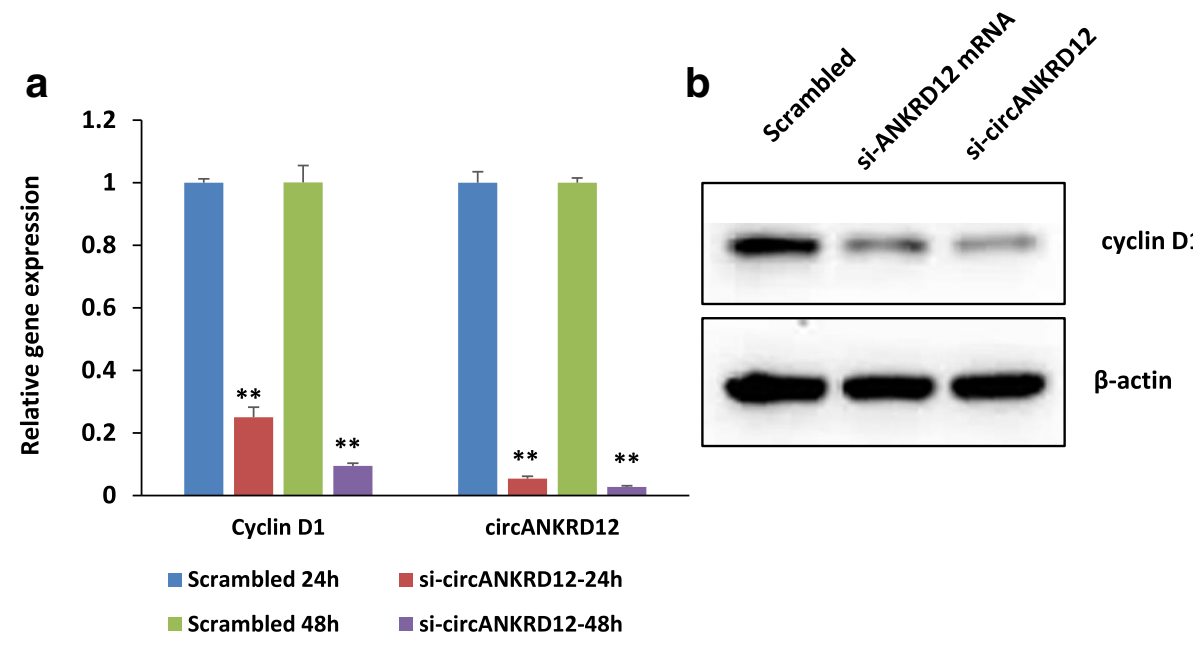

C

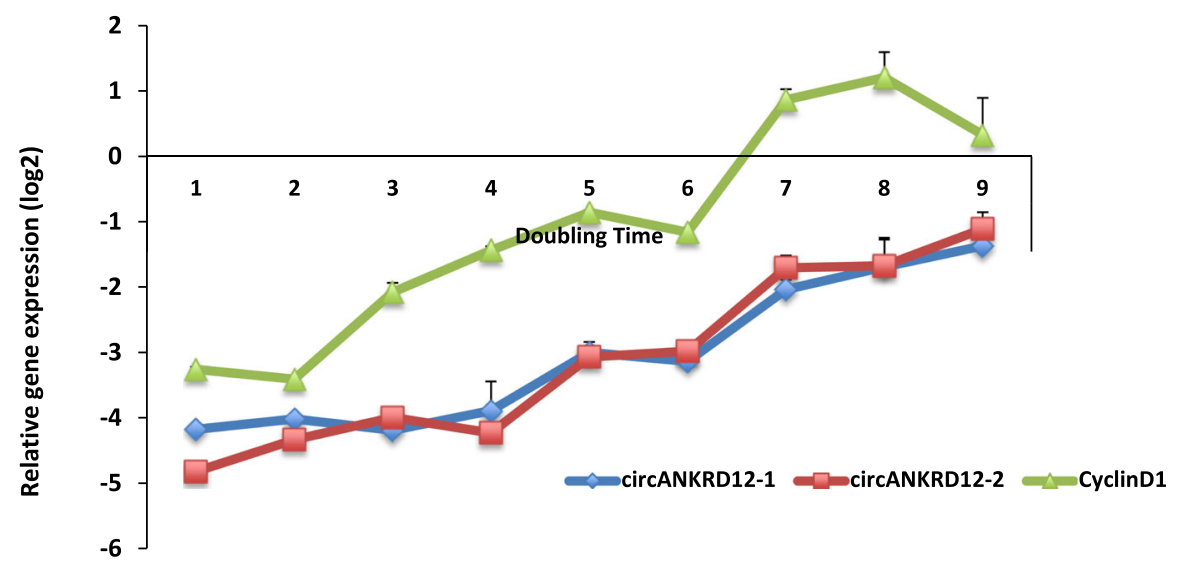

d

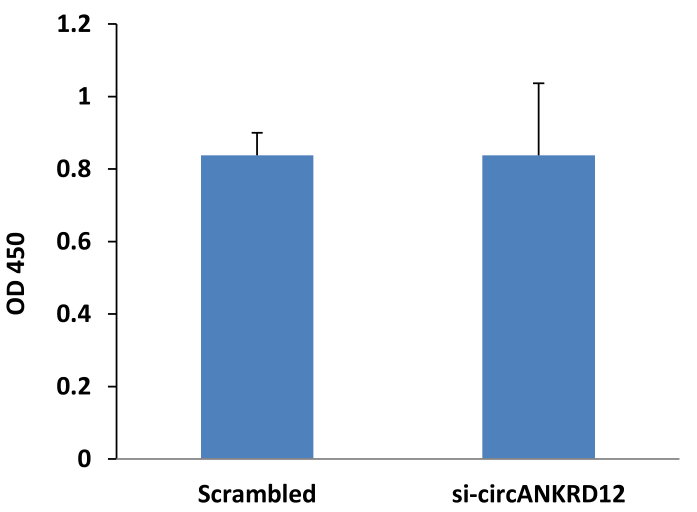

Fig. 7 Longevity of circANKRD12 silencing in SKOV3 cells a qRT-PCR analysis of circANKRD12 knockdown efficiency at two different time points ( $24 \mathrm{~h}, 48 \mathrm{~h}$ ). b Western blot analysis of cyclin D1 expression in circANKRD12 and ANKRD12 silenced SKOV3 cells at $48 \mathrm{~h}$. $\mathbf{c}$ Longevity analysis of circANKRD12 silenced SKOV3 cells. qRT-PCR analysis for the longevity of siRNA transfection based on knockdown efficiency of circANKRD12 in each doubling time (48 h). The figure shows the Knockdown efficiency of circANKRD12 on cyclin D1 expression. qRT-PCR of the expression pattern of cyclin D1 from 1st to 9th doubling time d MTS cell proliferation assay of circANKRD12 knockdown cells after 10th doubling. Data in a is the means with error bars representing standard error of the mean. ${ }^{*} P<0.01$ (Student's $t$-test) 

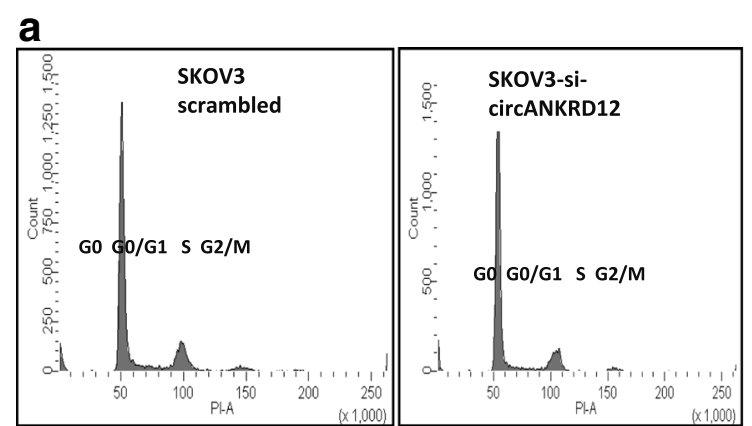

C

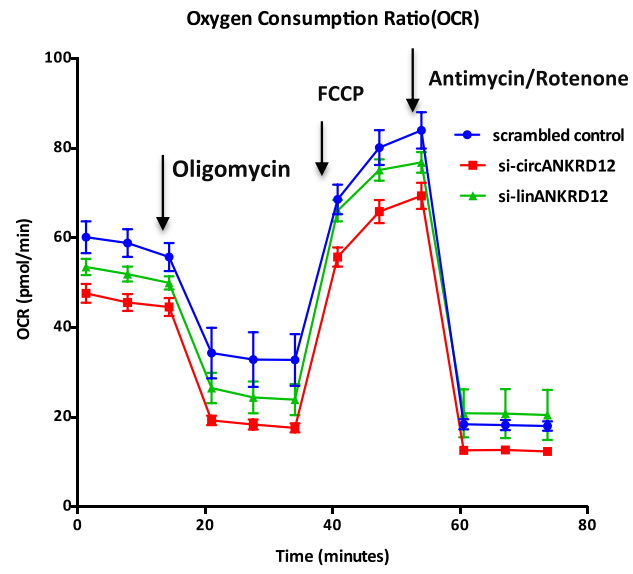

e

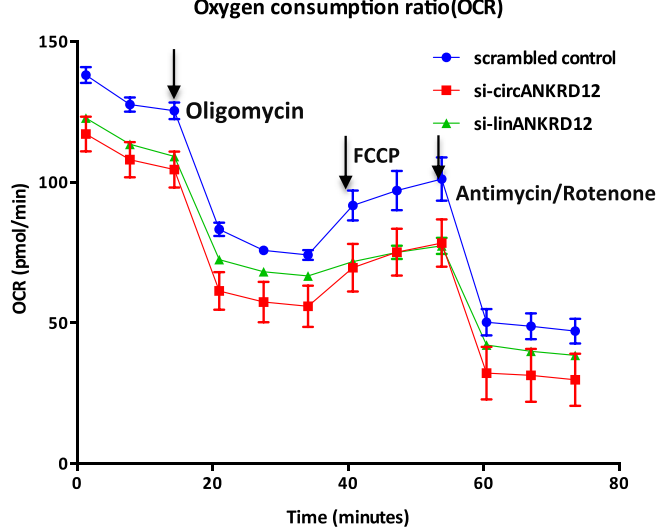

b

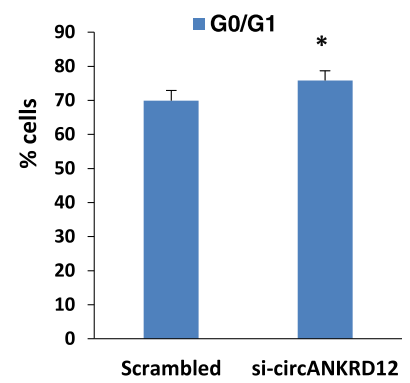

d

ATP linked OCR

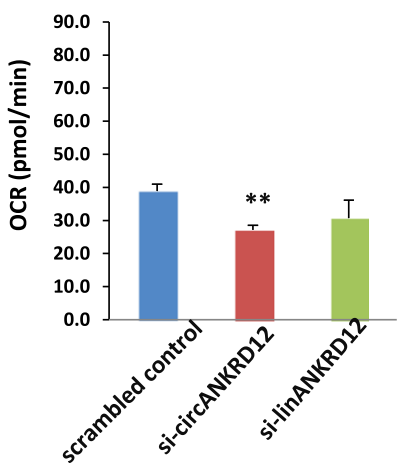

Fig. 8 circANKRD12 silenced cells have an arrested G1stage and low OXPHOS compared to scrambled control. a Cell cycle analysis of SKOV3 cells silenced with circANKRD12 at $24 \mathrm{~h}$. b Bar chart representation of \% of cells in G0/G1 stage. Error bars represent standard error of the mean from three experimental replicates. c,d Oxygen consumption ratio and ATP production analyzed by seahorse extracellular flux analyzer in MDAMB231 cells silenced with si-circANKRD12, si-linear ANKRD12 and its control. e Oxygen consumption ratio analyzed by seahorse by extracellular flux analysis in SKOV3 cells. Data in $\mathbf{c}$, $\mathbf{d}$, and $\mathbf{e}$ are normalized with total protein(ug/ul)

STAT-1, MX1, NFkB, MUC4, and SMAD3. Cell based assays live cell migration, invasion and wound healing assays also shows increased invasion of circANKRD12 silenced cells in SKOV3 cell line.

Cell proliferation was significantly arrested without any change in cell viability in circANKRD12 silenced SKOV3 cells. Cyclin D1, a consistently affected gene by circANKRD12 silencing, is down regulated thereby reducing cell proliferation and increasing invasion. Previous studies have reported a reduction in cyclin D1 levels, and G0/G1 cell cycle stage arrest leads to an increase in migratory activities of MDA-MB-231 breast cancer cells [36]. Consistent with these findings, our results of cell cycle analysis also show a substantial G0/G1 arrest with increased migration and invasion in circANKRD12 knockdown cells. The migration and 
invasion assays significantly correlate with gene expression analysis and indicate an augmented cell migration and invasion rate. The 3D anchorage independent organotypic tumor models of SKOV3 show similar patterns of phenotypic characterization where invasion through collagen is increased upon silencing of circANKRD12. There is a strong phenotypic alteration after silencing circANKRD12 in the ovarian cancer cells in both 2D and 3D culture conditions suggesting that circANKRD12 is important in regulating proliferation, invasion, and migration. Reduction in cell proliferation and regulation of Cyclin D1 expression is confirmed by silencing circANKRD12 by another construct of siRNA in SKOV3 cells (Additional file 3: S2:16a, b). circRNAs are involved in regulating cellular movement. Indeed, circRNA (F-circEA) produced from the EML4-ALK fusion gene which is independent of the EML4-ALK linear transcript and the fusion protein, can promote migration and invasion, thus contributing to tumor metastasis [37, 38].

The Ankyrin Repeat Domain family of genes can act as putative tumor suppressors via p53 mediated feedback or through recruiting histone deacetylases (HDACs) to the p160 coactivator to repress transcriptional activities $[18,19,39]$. A clinical study of gene expression of ANKRD12 in colorectal cancer revealed that low ANKRD12 expression is correlated with overall poor survival and liver metastasis of CRC patients [20].

We observed that the silencing of ANKRD12 mRNA reduces cell proliferation, induces cell death and down regulates cyclin D1. On the contrary, silencing of circANKRD12 arrests the cell cycle progression and increases tumor invasion without significantly affecting cell viability. The knockdown of circANKRD12 can change the oncobioenegetics as its shift from a higher OXPHOS to a lower OXPHOPS phenotype which is highly invasive. circANKRD12 may act as competing endogenous RNA (ceRNA) to regulate a circRNAmiRNA-mRNA network. Our insilco analysis shows that cyclin D1 and circANKRD12 have shared binding sites for several different microRNAs (Additional file 6: Table S6). Thus, circANKRD12 could act as a microRNA sponge to regulate cyclin D1 levels. Our preliminary analysis shows overexpression of hsa-miR-4768-5p reduces the level of Cyclin D1 by 20\% (hsa-miR-4768-5p has common binding sites for circANKRD12 and Cyclin D1) (Data not shown). Thus, circANKRD12 could act as a microRNA sponge to regulate cyclin D1 levels. We also observed that circANKRD12 contains some putative open reading frames (ORFs) and therefore its translation through internal ribosomal entry sites cannot be ruled out $[40,41]$.

\section{Conclusion}

In conclusion, our study provides the molecular, phenotypic and metabolic characterization of one of the most abundant circRNA in human ovarian and breast cancer cells. Our results suggest that circANKRD12 could be involved in a diverse set of functions ranging from cell cycle arrest, tumor invasion to immune modulation. Manipulating the levels of circANKRD12 can regulate molecular functions by altering different signaling pathways and modifies the phenotype of the cells. The distinctive change from a proliferative to a more invasive phenotype by altering circANKRD12 levels could lead to a future circRNA based therapeutic intervention in cancer.

\section{Additional files}

Additional file 1: Figure S1. (a) The abundance of circANKRD12 and ANKRD12 linear RNA. Semi qRT-PCR data indicating the abundance of circANKRD12 and ANKRD12 mRNA in the cytoplasmic and nuclear fractions of SKOV3 cells, nuclear and cytoplasmic purity markers were also assessed using nuclear specific marker 7SK and cytoplasmic specific marker CYTB. The gel picture represents PCR amplified products of circular RNAs with three different sets of primers. (b) Real-time data shows the abundance of circANKRD12 and ANKRD12 mRNA in the cytoplasm and nucleus. (c) The abundance of circANKRD12 and ANKRD12 mRNA in a panel of cancer cells. (PPTX 2728 kb) (PPTX $2750 \mathrm{~kb}$ )

Additional file 2: Figure S2. Figure $a, b, c$ Shows functional predicted networks from RNAseq data of circANKRD12 silenced MDA-MB-231 cells induces inflammatory immune responses and cancer cell invasion with activation Z score and p values. (PPTX 6928 kb) (PPTX 6949 kb)

Additional file 3: S2. File represents additional figures for validating the circANKRD12 in cell lines. (PPTX 17563 kb) (PPTX 17572 kb)

Additional file 4: Tables S1-S5. Tables representing primers used for different gene expression studies, siRNAs and pathways involved in circANKRD12 gene knockdown condition. (PPTX 3253 kb) (PPTX 3268 kb)

Additional file 5: Supplementary file S1. List of genes differentially expressed in circANKRD12 silenced cells compared to control in different cell lines. (XLSX $160 \mathrm{~kb})$

Additional file 6: Table S6. List of microRNAs that can target circANKRD12 and CyclinD1. (XLSX 9 kb)

\section{Abbreviations}

3D culture: Three-Dimensional cell culture; circRNA: Circular RNA; FDR: False discovery rate; OCR: Oxygen Consumption Ratio; OXPHOS: Oxidative Phosphorylation; qRT-PCR: Quantitative Real Time PCR; RNAseq: RNA sequencing; siRNA: Small (or short) interfering RNA

\section{Acknowledgments}

The work was supported by grants from Basic Medical Research Program (BMRP) grant from Qatar Foundation to WCM-Q.

We thank Shameem Yunuskunju form Genomics Core at WCM-Q for RNA -Seq data handling. We thank Dr. Anna Halama from Dr. Karsten Suhre's Bioinformatics Core lab at WCM-Q for providing the LL24, NCl-H226, T47D, HCC2935 cells. We also thank Ms. Aleksandra M. Liberska from Microscopy Core at WCM-Q for helping with Flow Cytometry.

\section{Funding}

This study was supported by Weill Cornell Medicine and Qatar Foundation (BMRP 1 - Malek Pilot FY17). The funders had no role in the design of the study, data analysis, interpretation of data and writing the manuscript.

\section{Availability of data and materials}

The datasets supporting the conclusions of this article are included in this article and the Supplementary Data.

\section{Authors' contributions}

JM, TK, and IA designed the research; TK, WAA, FAD, SA, and SS performed cellular experiments. TK and IA contributed equally to this work. IAA and 
YAM performed RNA-seq and IA and JM performed bioinformatic analysis; AR provided the cell lines and helped with experimental design; TK and IA wrote the manuscript. All authors read and approved the final manuscript.

\section{Ethics approval and consent to participate}

Not applicable for the present study. All experiments were conducted in cancer cell lines, no human or animal subject is involved in the study.

\section{Consent for publication}

Not applicable.

\section{Competing interests}

The authors declare that they have no competing interests.

\section{Publisher's Note}

Springer Nature remains neutral with regard to jurisdictional claims in published maps and institutional affiliations.

\section{Author details}

${ }^{1}$ Department of Genetic Medicine, Weill Cornell Medicine-Qatar, Education City, Qatar Foundation, P.O. Box No, 24144 Doha, Qatar. ${ }^{2}$ Genomics Core, Weill Cornell Medicine-Qatar, Education City, Qatar Foundation, Doha, Qatar. ${ }^{3}$ Department of Pharmacology, Weill Cornell Medicine-Qatar, Education City, Qatar Foundation, Doha, Qatar. ${ }^{4}$ Stem Cell and Microenvironment Laboratory, Weill Cornell Medicine-Qatar, Education City, Qatar Foundation, Doha, Qatar.

\section{Received: 25 July 2018 Accepted: 23 April 2019}

\section{Published online: 11 June 2019}

\section{References}

1. Jeck WR, Sharpless NE. Detecting and characterizing circular RNAs. Nat Biotechnol. 2014;32. https://doi.org/10.1038/nbt.2890.

2. Cocquerelle C, Mascrez B, Hétuin D, Bailleul B. Mis-splicing yields circular RNA molecules. FASEB J. 1993;7:155-60 https://www.fasebj.org/doi/abs/10. 1096/fasebj.7.1.7678559. Accessed 1 May 2017.

3. Wang PL, Bao Y, Yee M-C, Barrett SP, Hogan GJ, Olsen MN, et al. Circular RNA is expressed across the eukaryotic tree of life. PLoS One. 2014;9:e90859. https://doi.org/10.1371/journal.pone.0090859.

4. Dang Y, Yan L, Hu B, Fan X, Ren Y, Li R, et al. Tracing the expression of circular RNAs in human pre-implantation embryos. Genome Biol. 2016. https://doi.org/10.1186/s13059-016-0991-3.

5. Hansen $T B$, Jensen $T I$, Clausen BH. Natural RNA circles function as efficient microRNA sponges. Nature. 2013;495. https://doi.org/10.1038/nature11993.

6. Ashwal-Fluss R, Meyer M, Pamudurti NR, Ivanov A, Bartok O, Hanan M, et al. circRNA biogenesis competes with pre-mRNA splicing. Mol Cell. 2014;56. https://doi.org/10.1016/j.molcel.2014.08.019.

7. Li J, Yang J, Zhou P, et al. Circular RNAs in cancer: novel insights into origins, properties, functions and implications. Am J Cancer Res. 2015;5(2):472-80.

8. He J, Xie Q, Xu H, Li J, Li Y. Circular RNAs and cancer. Cancer Lett. 2017;396: 138-44. https://doi.org/10.1016/j.canlet.2017.03.027.

9. Qu S, Liu Z, Yang X, Zhou J, Yu H, Zhang R, et al. The emerging functions and roles of circular RNAs in cancer. Cancer Lett. 2018;414:301-9. https://doi. org/10.1016/J.CANLET.2017.11.022.

10. Li Z, Yanfang W, Li J, Jiang P, Peng T, Chen K, et al. Tumor-released exosomal circular RNA PDE8A promotes invasive growth via the miR-338/ MACC1/MET pathway in pancreatic cancer. Cancer Lett. 2018. https://doi. org/10.1016/j.canlet.2018.04.035.

11. Chen B, Huang S. Circular RNA: An emerging non-coding RNA as a regulator and biomarker in cancer. Cancer Lett. 2018;418:41-50. https://doi. org/10.1016/j.canlet.2018.01.011.

12. Meng X, Li X, Zhang P, Wang J, Zhou Y, Chen M. Circular RNA: an emerging key player in RNA world. Brief Bioinform. 2016:bbw045. https://doi.org/10. 1093/bib/bbw045

13. Memczak S, Papavasileiou P, Peters O, Rajewsky N. Identification and characterization of circular RNAs as a new class of putative biomarkers in human blood. PLoS One. 2015;10:e0141214. https://doi.org/10.1371/journal. pone.0141214

14. Hang D, Zhou J, Qin N, Zhou W, Ma H, Jin G, et al. A novel plasma circular RNA circFARSA is a potential biomarker for non-small cell lung cancer. Cancer Med. 2018. https://doi.org/10.1002/cam4.1514.
15. Xia W, Qiu M, Chen R, Wang S, Leng X, Wang J, et al. Circular RNA has_circ_ 0067934 is upregulated in esophageal squamous cell carcinoma and promoted proliferation. Sci Rep. 2016;6:35576. https://doi.org/10.1038/srep35576.

16. Li F, Zhang L, Li W, Deng J, Zheng J, An M, et al. Circular RNA ITCH has inhibitory effect on ESCC by suppressing the Wnt $/ \$ \beta \$$-catenin pathway. Oncotarget. 2015;6:6001-13. https://doi.org/10.18632/oncotarget.3469.

17. Dong Y, He D, Peng Z, Peng W, Shi W, Wang J, et al. Circular RNAs in cancer: an emerging key player. J Hematol Oncol. 2017. https://doi.org/10. 1186/s13045-016-0370-2

18. Li C-W, Dinh GK, Zhang A, Chen JD. Ankyrin repeats-containing cofactors interact with ADA3 and modulate its co-activator function. Biochem J. 2008. https://doi.org/10.1042/BJ20071484.

19. Neilsen PM, Cheney KM, Li C-W, Chen JD, Cawrse JE, Schulz RB, et al. Identification of ANKRD11 as a p53 coactivator. J Cell Sci. 2008;121 https:// www.ncbi.n/m.nih.gov/pubmed/?term=Identification+of+ANKRD11+as+a+ p53+coactivator.+J+Cell+Sci.+2008. Accessed 11 Apr 2017.

20. Bai R, Li D, Shi Z, Fang X, Ge W, Zheng S. Clinical significance of Ankyrin repeat domain 12 expression in colorectal cancer. J Exp Clin Cancer Res. https://doi.org/10.1186/1756-9966-32-35.

21. Ahmed I, Karedath T, Andrews SS, Al-Azwani IK, Ali Mohamoud Y, Querleu $D$, et al. Altered expression pattern of circular RNAs in primary and metastatic sites of epithelial ovarian carcinoma. Oncotarget. 2016. https:// doi.org/10.18632/oncotarget.8917.

22. Zheng Q, Bao C, Guo W, Li S, Chen J, Chen B, et al. Circular RNA profiling reveals an abundant circHIPK3 that regulates cell growth by sponging multiple miRNAs. Nat Commun. 2016;7:11215. https://doi.org/10.1038/ncomms11215.

23. Liang C-C, Park AY, Guan J-L. In vitro scratch assay: a convenient and inexpensive method for analysis of cell migration in vitro. Nat Protoc. 2007; 2:329-33. https://doi.org/10.1038/nprot.2007.30.

24. Luo J, Wang X, Xia Z, Yang L, Ding Z, Chen S, et al. Transcriptional factor specificity protein 1 (SP1) promotes the proliferation of glioma cells by upregulating midkine (MDK). Mol Biol Cell. 2015;26:430-9. https://doi.org/10. 1091/mbc.E14-10-1443.

25. Samuel SM, Ghosh S, Majeed Y, Arunachalam G, Emara MM, Ding H, et al. Metformin represses glucose starvation induced autophagic response in microvascular endothelial cells and promotes cell death. Biochem Pharmacol. 2017;132:118-32. https://doi.org/10.1016/J.BCP.2017.03.001.

26. Vincent HA, Deutscher MP. Substrate recognition and catalysis by the exoribonuclease RNase R. J Biol Chem. 2006;281:29769-75. https://doi.org/ 10.1074/jbc.M606744200.

27. Pampaloni F, Reynaud EG, Stelzer EHK. The third dimension bridges the gap between cell culture and live tissue. Nat Rev Mol Cell Biol. 2007:8:839-45. https://doi.org/10.1038/nrm2236.

28. Baldin V, Lukas J, Marcote MJ, Pagano M, Draetta G. Cyclin D1 is a nuclear protein required for cell cycle progression in G1. Genes Dev. 1993;7:812-21. https://doi.org/10.1101/GAD.7.5.812.

29. Yang L, Moss T, Mangala LS, Marini J, Zhao H, Wahlig S, et al. Metabolic shifts toward glutamine regulate tumor growth, invasion and bioenergetics in ovarian cancer. Mol Syst Biol. 2014;10. https://doi.org/10.1002/msb.

30. Vayalil PK, Landar A. Mitochondrial oncobioenergetic index: a potential biomarker to predict progression from indolent to aggressive prostate cancer. Oncotarget. 2015;6:43065-80. https://doi.org/10.18632/oncotarget.5487.

31. Guarnerio J, Bezzi M, Jeong JC, Paffenholz SV, Berry K, Naldini MM, et al. Oncogenic role of fusion-circRNAs derived from Cancer-associated chromosomal translocations. Cell. 2016;165:289-302. https://doi.org/10.1016/j.cell.2016.03.020.

32. Du WW, Yang W, Chen Y, Wu Z-K, Foster FS, Yang Z, et al. Foxo3 circular RNA promotes cardiac senescence by modulating multiple factors associated with stress and senescence responses. Eur Heart J. 2016:ehw001. https://doi.org/10.1093/eurheartj/ehw001.

33. Huang G, Zhu H, Shi Y, Wu W, Cai H, Chen X, et al. Cir-ITCH plays an inhibitory role in colorectal Cancer by regulating the $\mathrm{Wnt} / \$ \beta \$$-catenin pathway. PLoS One. 2015;10:e0131225. https://doi.org/10.1371/journal.pone.0131225.

34. Dong W, Bi J, Liu H, Yan D, He Q, Zhou Q, et al. Circular RNA ACVR2A suppresses bladder cancer cells proliferation and metastasis through miR626/EYA4 axis. https://doi.org/10.1186/s12943-019-1025-z.

35. Nicolet BP, Engels $\mathrm{S}$, Aglialoro $\mathrm{F}$, van den Akker $\mathrm{E}$, von Lindern $\mathrm{M}$, Wolkers MC. Circular RNA expression in human hematopoietic cells is widespread and cell-type specific. Nucleic Acids Res. 2018;46:8168-80. https://doi.org/10. 1093/nar/gky721.

36. Lehn S, Tobin NP, Berglund P, Nilsson K, Sims AH, Jirström K, et al. Downregulation of the oncogene cyclin D1 increases migratory capacity in breast 
cancer and is linked to unfavorable prognostic features. Am J Pathol. 2010; 177:2886-97. https://doi.org/10.2353/ajpath.2010.100303.

37. Tan S, Gou Q, Pu W, Guo C, Yang Y, Wu K, et al. Circular RNA F-circEA produced from EML4-ALK fusion gene as a novel liquid biopsy biomarker for non-small cell lung cancer. Cell Res. 2018;28:693-5. https://doi.org/10. 1038/s41422-018-0033-7.

38. Tan S, Sun D, Pu W, Gou Q, Guo C, Gong Y, et al. Circular RNA F-circEA-2a derived from EML4-ALK fusion gene promotes cell migration and invasion in non-small cell lung cancer. Mol Cancer. 2018;17:138. https://doi.org/10. 1186/s12943-018-0887-9.

39. Zhang A, Li C-W, Chen JD. Characterization of transcriptional regulatory domains of ankyrin repeat cofactor-1. Biochem Biophys Res Commun. 2007; 358:1034-40. https://doi.org/10.1016/j.bbrc.2007.05.017.

40. Legnini I, Di Timoteo G, Rossi F, Morlando M, Briganti F, Sthandier O, et al. CircZNF609 is a circular RNA that can be translated and functions in Myogenesis. Mol Cell. 2017;66:22-37.e9. https://doi.org/10.1016/j.molcel.2017.02.017.

41. Pamudurti NR, Bartok O, Jens M, Ashwal-Fluss R, Stottmeister C, Ruhe L, et al. Translation of CircRNAs. Mol Cell. 2017;66:9-21.e7. https://doi.org/10. 1016/j.molcel.2017.02.021.

Ready to submit your research? Choose BMC and benefit from:

- fast, convenient online submission

- thorough peer review by experienced researchers in your field

- rapid publication on acceptance

- support for research data, including large and complex data types

- gold Open Access which fosters wider collaboration and increased citations

- maximum visibility for your research: over $100 \mathrm{M}$ website views per year

At $\mathrm{BMC}$, research is always in progress.

Learn more biomedcentral.com/submissions 